doi:10.17659/01.2019.0038

Journal of Case Reports 2019;9(3):146-148

\title{
Functional Adrenal Adenoma Presenting as Ventricular Tachycardia in a Young Boy
}

\author{
S Anuradha, Ayush Mohan Makkar, Sohini Haldar, RS Ahlawat \\ Department of Medicine, Maulana Azad Medical College, Bahadur Shah Zafar Marg, New Delhi-110002, India.
}

\begin{abstract}
Corresponding Author:
Dr. S. Anuradha

Email: drsanuradha@gmail.com

This is an Open Access article distributed under the terms of the Creative Commons Attribution License (creativecommons.org/ licenses/by/3.0).

Received : December 26, 2018

Accepted : June 18,2019

Published : July 15, 2019
\end{abstract}

\begin{abstract}
Background: Ventricular tachycardia may be a solitary episode, complication of a structural heart disease or unusually, the manifestation of a serious electrolyte abnormality. Case Report: We present the rare case of a 13-year-old boy who presented with sudden onset ventricular tachycardia that was precipitated by severe hypokalemia. Subsequently, the associated hypertension and metabolic alkalosis prompted a search for an underlying endocrine abnormality. After investigations, the patient was diagnosed as a case of Conn's syndrome due to a functional adrenal adenoma- a rare diagnosis. Conclusion: The initial presentation of hyperaldosteronism in a child as ventricular tachycardia is extremely rare.
\end{abstract}

Keywords: Adrenocortical Adenoma, Alkalosis, Hyperaldosteronism, Hypokalemia, Ventricular Tachycardia.

\section{Introduction}

Primary hyperaldosteronism is a syndrome classically characterized by hypertension, hypokalemia, elevated levels of serum aldosterone, and consistently suppressed levels of plasma renin activity $[1,2]$. Here we present the rare case of an adolescent, eventually diagnosed as Conn's syndrome, presenting with severe hypokalemia leading to ventricular tachycardia. There are very few reports of cases of Conn's syndrome presenting as ventricular tachycardia and to the best of our knowledge this is first report describing such a case in adolescents.

\section{Case Report}

A 13 year old adolescent male presented to the medical emergency in an unconscious state after sudden collapse at home. On examination, his peripheral pulses were absent and blood pressure was non-recordable. The cardiac examination was unremarkable. An electrocardiogram showed wide complex polymorphic ventricular tachycardia.
The patient was resuscitated using standard ACLS protocols and was shifted to the Intensive Care Unit. An arterial blood gas analysis and serum electrolytes were obtained which revealed metabolic alkalosis and severe hypokalemia (serum potassium $2.1 \mathrm{meq} / \mathrm{L})$. After initial resuscitation, a thorough history was taken and detailed examination performed. There was a history of intermittent pain in bilateral lower limbs along with polyuria and polydipsia for last one year. Physical examination was unremarkable. Sinus rhythm was achieved and maintained and management for hypokalemia was instituted.

During the patient's stay in the hospital, his blood pressure was elevated on multiple recordings (140/90 $\mathrm{mm} \mathrm{Hg})$. He had persistent hypokalemia (serum potassium 2.1-2.3 meq/L) and metabolic alkalosis which was refractory to conservative corrective measures. The patient was started on mineralocorticoid antagonists, tablet spironolactone, in view of hypertension associated with hypokalemia and he was investigated for the 
cause. An ultrasound of the abdomen revealed a heteroechoic mass lesion in the upper pole of left kidney $(4.5 \times 3.8 \mathrm{~cm})$ suggestive of an adrenal mass. The kidneys were of normal size and echogenicity. Renal artery Doppler was performed and the renovascular causes of hypertension were ruled out. The rest of the biochemical parameters were normal: serum creatinine $0.7 \mathrm{mg} / \mathrm{dL}$, blood urea 28 $\mathrm{mg} / \mathrm{dL}$, blood sugar $104 \mathrm{mg} / \mathrm{dL}$, serum sodium 137 meq/L, serum calcium $9.6 \mathrm{mg} / \mathrm{dL}$, TSH $3.7 \mathrm{mIU} / \mathrm{L}$. A contrast enhanced CT scan of the abdomen demonstrated a well-defined, marginated enhancing soft tissue lesion involving the left adrenal gland of size $4.5 \times 3.9 \mathrm{~cm}$. The lesion showed a delayed washout suggestive of a benign adrenal adenoma [Fig.1]. An MRI abdomen confirmed a lesion $3.9 \times 4.2 \times 3.8 \mathrm{~cm}$ in the left adrenal gland which appeared hypointense on $\mathrm{T} 1 \mathrm{~W}$, hyperintense on T2W images and showed signal drop on opposed phase imaging likely to represent functional adrenal adenoma. A serum aldosterone level could not be done as it was not available.

In view of the functional adrenal adenoma, the patient underwent a laparoscopic adrenalectomy and the adenoma was removed [Fig.2]. The biopsy of the tumor was suggestive of an adrenal cortical adenoma with no areas of necrosis. One week after surgery, spironolactone was stopped and the blood pressure and hypokalemia normalized. A final diagnosis of adrenal adenoma with primary hyperaldosteronism leading to hypokalemia induced ventricular tachycardia was made.

\section{Discussion}

Primary hyperaldosteronism caused by adrenal adenoma is predominantly a disease of adults. In children, the clinical syndrome of hyperaldosteronism, hypertension, and hyporeninemia has rarely been attributed to a solitary adrenal adenoma (Conn syndrome). Ventricular arrhythmia in association with primary hyperaldosteronism is an uncommon presentation that has previously been described in the literature

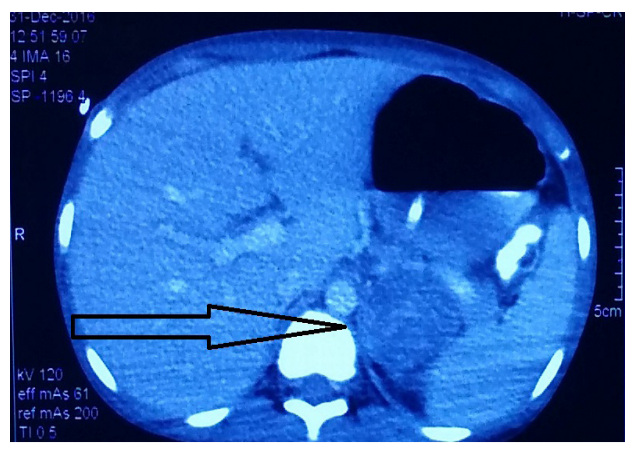

Fig.1: Contrast enhanced CT scan of the abdomen demonstrating a well-defined, marginated enhancing soft tissue lesion involving the left adrenal gland of size $4.5 \times 3.9 \mathrm{~cm}$ (arrow).

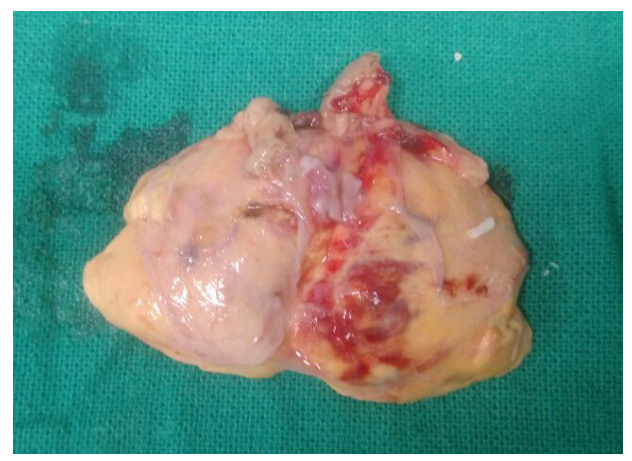

Fig.2: The adrenal adenoma removed at surgery.

in isolated reports [3-6]. Prolongation of QT interval due to hypokalemia and aldosterone excess may result in the development of life-threatening arrhythmia such as torsade de pointes or ventricular tachycardia [5]. Our report describes an adolescent presenting with hypokalemia induced ventricular arrhythmia due to Conn's syndrome. To the best of our knowledge, this is the first case report highlighting such an association in the pediatric age group.

Patients with primary hyperaldosteronism usually report fatigue, muscle weakness, cramps, headaches and palpitations. They can also have polydipsia and polyuria from hypokalemia induced nephrogenic diabetes insipidus. Long standing hypertension may lead to cardiac, retinal, renal and neurological problems, with all the associated symptoms and signs. Screening tests for primary 
hyperaldosteronism include the serum potassium and bicarbonate levels and plasma aldosterone/ plasma renin activity ratio. Confirmatory tests include serum aldosterone level and 24 hour urinary aldosterone excretion test. The initial radiologic investigation in the work up of primary hyperaldosteronism is high resolution, thin sliced (2-2.5 $\mathrm{mm}$ ) adrenal computed tomography (CT) scanning with contrast. Pharmacological therapy includes calcium channel blockers, mineralocorticoid antagonists. Surgery is the treatment of choice for typical aldosteronomas. An adrenalectomy can be performed via a formal laparotomy or by using a laparoscopic technique.

\section{Conclusion}

The detection of hypokalemia, metabolic alkalosis and subsequent hypertension in an adolescent raised the diagnostic suspicion of an underlying endocrinological disorder. All subjects who present with unexplained ventricular arrhythmia should be investigated to rule out an underlying clinical disorder.
Contributors: SA: manuscript editing, patient management; AMM, SH: manuscript writing, patient management; RSA: critical inputs into the manuscript. SA will act as guarantor. All authors approved the final version of this manuscript.

Funding: None; Competing interests: None stated.

\section{References}

1. Baranwal AK, Singhi SC, Narshimhan KL, Jayashree M, Singhi PD, Kakkar N. Aldosterone-producing adrenocortical adenoma in childhood: a case report. J Pediatr Surg. 1999;34:1878-1880.

2. Bryer-Ash M, Wilson DM, Tune BM, Rosenfeld RG, Shochat SJ, Luetscher JA. Hypertension caused by an aldosterone secreting adenoma. Occurence in a 7-yearold child. Am J Dis Child. 1984;138:673-676.

3. Zelinka T, Holaj R, Petrak O, Kasalický M, Hanus T, et al. Life-threatening arrhythmia caused by primary aldosteronism. Med Sci Monit. 2009;15:CS174-177.

4. Sade E, Oto A, Oner Z, Daver A, Onalan O, Bilezikci $\mathrm{B}$, et al. Adrenal adenoma presenting with torsade de pointes-a case report. Angiology. 2002;53:471-474.

5. Abdo A, Bebb RA, Wilkins GE. Ventricular fibrillation: an extreme presentation of primary hyperaldosteronism. Can J Cardiol. 1999;15:347-348.

6. Higham PD, Adams PC, Murray A, Campbell RW. Plasma potassium, serum magnesium and ventricular fibrillation: a prospective study. Q J Med. 1993;86:609-617. 